\title{
La escuela es territorio. Cartografía social de experiencias pedagógicas en instituciones educativas de Medellín y Bello, Colombia
}

The School is a Territory. Social Mapping of Pedagogical Experiences in Public Schools of Medellín and Bello, Colombia

A escola é território. Mapeamento social de experiências pedagógicas em instituições de ensino de Medellín e Bello, na Colômbia

César Andrés Ospina Mesa*

Vladimir Montoya Arango* Lida Sepúlveda López ${ }^{* * *}$

Recibido: 30 de abril del 2020

Aprobado: 24 de noviembre del 2020

https://doi.org/10.12804/revistas.urosario.edu.co/territorios/a.9024

Para citar este artículo:

Ospina Mesa, C., Montoya Arango, V., \& Sepúlveda López, L. (2021). La escuela es territorio. Cartografía social de experiencias pedagógicas en instituciones educativas de Medellín y Bello, Colombia. Territorios, (44-Especial), 15-34. https://doi.org/10.12804/revistas.urosario.edu.co/territorios/a.9024

* Universidad de Antioquia, Instituto de Estudios Regionales, Medellín, Colombia. Correo electrónico: cesara.ospina@ udea.edu.co ORCID: bttps://orcid.org/00000002-5879-1509

** Universidad de Antioquia, Instituto de Estudios Regionales, Medellín, Colombia. Correo electrónico: vladimir.montoya@udea.edu.co ORCID: https://orcid.org/00000002-8000-9405

*** Universidad de Antioquia, Instituto de Estudios Regionales Medellin, Colombia. Correo electrónico: lida. sepulveda@udea.edu. co ORCID: https://orcid. org/0000-0003-34618979 
Palabras clave

Escuela; territorio; experiencias

pedagógicas; cartografía social; Colombia.

Keywords

School; territory; pedagogical experiences; social cartography; school; Colombia.

Palavras-chave

Escola; território; experiências pedagógicas; mapeamento social; Colômbia.

\section{territarias 44 Especial}

\section{RESUMEN}

La escuela es un territorio producto de complejas relaciones históricas y geográficas de la diversidad de personas que la habitan. Entre el 2013 y el 2016 se llevaron a cabo dos proyectos de investigación en instituciones educativas de las ciudades de Medellín y Bello (Antioquia), articulados en torno a la noción de pedagogía del territorio, en los que se empleó la cartografía social y la etnografía como estrategia de construcción colectiva de conocimientos territoriales con la participación de maestros, estudiantes y directivos, en búsqueda de dilucidar las maneras en las cuales la escuela se constituye en un lugar donde se producen conocimientos asociados a pedagogías y metodologías específicas que vinculan a los actores sociales de los territorios en los que ella se sitúa, poniendo en tensión discursos y prácticas que representan la escuela como un lugar dedicado únicamente a la reproducción de información y conocimientos preestablecidos.

\section{ABSTRACT}

The school is a territory product of complex historical and geographical relationships of the diversity of people who inhabit it. Between 2013 and 2016, two research projects were carried out in educational institutions in the cities of Medellín and Bello (Antioquia), articulated around the notion of a pedagogy of the territory, in which social cartography and ethnography were used as strategies collective construction of territorial knowledge with the participation of teachers, students, and managers, seeking to elucidate how the school is constituted in a place where knowledge associated with specific pedagogies and methodologies that articulate the social actors of the territories in those that she is located, putting in tension discourses and practices that represent the school as a place dedicated solely to the reproduction of preestablished information and knowledge.

\section{RESUMO}

A escola é um território que é produto de complexas relações históricas e geográficas da diversidade de pessoas que a habitam. Entre 2013 e 2016, foram realizados dois projetos de pesquisa em instituições de ensino das cidades de Medellín e Bello (Antioquia), articulados em torno da noção de pedagogia do território, em que o mapeamento social e a etnografia foram utilizadas como estratégias de construção coletiva de conhecimento territorial com a participação de professores, alunos e gestores, buscando elucidar os modos como a escola se constitui em um lugar onde se produzem saberes associados à pedagogias e metodologias específicas que articulam os atores sociais dos territórios onde se situa, tensionando discursos e práticas que representam a escola como um lugar dedicado exclusivamente à reprodução de informações e conhecimentos preestabelecidos. 


\section{Introducción}

La escuela es una institución compleja cargada de los múltiples sentidos que le confieren aquellos que la integran, a la vez, es productora de diversos significantes que se vinculan a sus experiencias y entran a ser parte de las dinámicas que dotan de sentido los espacios que habitan fuera de la escuela. De este modo, la escuela trasciende en mucho de su condición de edificio enclavado en un territorio específico, para convertirse en un dispositivo de inscripción de experiencias y generador de relaciones que son parte estructurante de los mecanismos mediante los cuales el espacio social se articula, se dota de sentidos, reproduce la memoria colectiva y alimenta la imaginación del porvenir. Esto es advertido por el maestro Paulo Freire cuando agudamente declara:

Si tuviéramos claro que fue aprendiendo como percibimos que es posible enseñar, entenderíamos con facilidad la importancia de las experiencias informales en las calles, en las plazas, en el trabajo, en los salones de clase de las escuelas, en los patios de los recreos, donde diferentes gestos de los alumnos, del personal administrativo, del personal docente, se cruzan llenos de significación. Hay una naturaleza testimonial en los espacios tan lamentablemente relegados de las escuelas (Freire, 2012, p. 44).

Por lo tanto, es imprescindible reconocer que en los procesos de enseñanza- aprendizaje, las experiencias que desde el entorno circundante son traídas al interior de la escuela constituyen elementos significativos sobre los que discurre la vida escolar. Además, el espacio en el que se localiza la escuela, así como las materialidades de las que se compone y que la rodean, se convierten también en agentes importantes para la generación de contenidos y experiencias que alimentan el proceso de enseñanza-aprendizaje, tal y como lo puntualiza Freire: "Hay una pedagogicidad indiscutible en la materialidad del espacio" (Freire, 2012, p. 45).

Lo anterior sirve de marco para la reflexión central que se desarrollará en este artículo, relacionada con el interés por reivindicar las formas particulares en las que la escuela produce conocimientos de cara a las tensiones y potencialidades propias de las realidades sociales, políticas y culturales que la atraviesan, lo cual fue el eje de reflexión/acción en dos proyectos de investigación adelantados desde el Grupo Estudios del Territorio adscrito al Instituto de Estudios Regionales de la Universidad de Antioquia.

El primer proyecto se denominó $L a$ escuela por dentro, realizado entre octubre del 2013 y junio del 2014, con la financiación de la Secretaría de Educación del municipio de Medellín, participaron 112 maestros y maestras de 22 instituciones educativas de la ciudad de Medellín, ubicadas en las comunas: Villa Hermosa, Doce de Octubre, Robledo, La América, Belén, Popular, Castilla, Buenos Aires y tersitorias 44 Especial 
Manrique, y, en los cinco corregimientos del área rural: Santa Elena, San Cristóbal, San Antonio de Prado, San Sebastián de Palmitas y Altavista. El segundo proyecto se llamó Apropiación social del territorio y educación para la sostenibilidad, el cual era parte del "Proceso de construcción de confianza y fortalecimiento institucional en el sector de La Gabriela, área de influencia de las plantas de agregados de Argos", realizado en el municipio de Bello (Antioquia) en el 2016, con la financiación de la empresa Argos y la Universidad de Antioquia. Participaron 20 maestros y maestras, 16 estudiantes y 14 madres de familia de la Institución Educativa La Gabriela.

Las investigaciones apuntaron a considerar la escuela desde su espacio físico y social como un territorio, esto es, como un entramado de relaciones de la comunidad educativa que la vive con una perspectiva esperanzadora, en tanto institución que aporta a la lucha por las dignidades y el respeto a la vida de maestros, maestras, estudiantes y familias. Los énfasis puestos en la escuela-territorio y en la escuela por dentro, propician la emergencia del concepto de pedagogía del territorio que apuntala la reflexión sobre los aprendizajes que desde las experiencias pedagógicas se vivencian en la escuela e irradian como elementos dinamizadores del territorio, mientras que este produce también reapropiaciones conceptuales que aleccionan a la escuela e inciden en sus prácticas curriculares. Se trata entonces de una relación en doble vía, en la cual la escuela favorece con su pedagogía la gestión de conflictos comunitarios incidiendo en el logro de territorios incluyentes, mientras que estos influyen en la democratización de la escuela. De esta manera, se argumenta que las prácticas educativas no son repeticiones incansables, sino que guardan estrecha vinculación con las maneras en que sucede la relación y el encuentro con el otro, entre maestros y estudiantes, familias y directivos, así como también con los procesos históricos y culturales de los barrios donde se encuentran ubicadas las escuelas.

Ahora bien, en el ámbito metodológico la exploración de la pedagogía del territorio en las escuelas implica el desafío de implementar estrategias adecuadas para aproximarse a los conocimientos locales y a la imaginación geográfica propia de los integrantes de la comunidad escolar, con el fin de integrar técnicas y métodos de producción colectiva de conocimiento que permita valorar y reconocer las experiencias pedagógicas en su contexto. La cartografía social es una estrategia metodológica pertinente para estimular y promover diálogos y, potenciar el intercambio de memorias, intereses y expectativas. En clave de una pedagogía del territorio, la cartografía social no solamente muestra su potencia como herramienta metodológica, sino que resulta esencial como estrategia de producción colaborativa de conocimientos sobre el territorio. Esto ratifica aplicaciones de la cartografía 
social al ámbito escolar referenciadas por autores diversos en América Latina, que han señalado el potencial pedagógico de esta metodología (Barragán et al., 2020; Parra, 2020; López, 2018; Uribe et al., 2017; Barragán, 2016), tal y como lo concluye Olga Patiño (2017) en su estudio sobre la aplicación al abordaje de problemas socioambientales y cuestiones socialmente vivas: "Se puede afirmar que la cartografía social, como medio o herramienta didáctica fortalece y renueva los procesos de enseñanza y de aprendizaje, aplicable no solo en la enseñanza de la geografía y las ciencias sociales sino en cualquier área de enseñanza" (Patiño, 2017, p. 164). En consecuencia, en los dos proyectos de investigación referidos, la cartografía social posibilitó la localización de saberes y experiencias que tienden a difuminarse y permanecer en el anonimato, haciendo que para la construcción colectiva de mapas, relatos y piezas audiovisuales, se generaran escenarios propicios para el autorreconocimiento, valoración de la labor pedagógica y del sentido humano de la educación escolar. Así, asumiendo las experiencias pedagógicas desarrolladas por los maestros en las aulas como unidad de análisis, se integraron en el proceso de construcción de las cartografías sociales tanto aquellas condiciones adversas que convergen al interior de la escuela, como algunas circunstancias que pueden favorecer la convivencia revelando el potencial que tiene la escuela como "espacio de contradiscurso" frente a las diversas realidades que viven estudiantes, maestros y, en general, la comunidad educativa con respecto a sus territorios consuetudinarios.

Tras una introducción en la que se describió el marco analítico general del presente trabajo, en este artículo se incluyó un segundo apartado en el que se analiza el uso de la cartografía social como una estrategia comprensiva de la escuela-territorio y la forma en que fue adaptada en los dos proyectos de investigación analizados; un tercer apartado en el que se vinculan las reflexiones sobre experiencias pedagógicas y territorio, desde los resultados y aprendizajes del acompañamiento a las instituciones educativas de las ciudades de Medellín y Bello; un cuarto apartado en el que se recogen algunas de las experiencias pedagógicas desde las voces de maestros y maestras y, finalmente, un quinto apartado en el que se exponen algunas conclusiones que recogen aprendizajes para una pedagogía del territorio desde las experiencias pedagógicas vividas en las escuelas y los barrios con los integrantes de las comunidades educativas.

\section{La cartografía social como estrategia comprensiva de la escuela-territorio}

La cartografía social se ha mostrado en los últimos años como una potente herramienta para explorar las percepciones territoriales de las comunidades locales y territarias 44 Especial 


\section{territorias} 44 Especial plasmarlas en mapas que luego se convierten en herramientas de lucha por el territorio (Oslender, 2017). También ha sido considerada como proceso y producto de conocimientos situados elaborados de manera dialógica y colaborativa, que parte por reconocer la relación estrecha entre espacio, poder y conocimiento, con lo cual se promueve la apertura metodológica a formas diversas de experimentar los territorios (Montoya et al., 2014). Otros autores en América Latina como Acselrad y Régis (2008), señalan que los procesos de mapeo participativo se han convertido en herramientas prácticas para el fortalecimiento de la movilización social, ya que favorecen las estrategias de control territorial y organización colectiva, además, son funcionales para la transmisión de las visiones comunitarias hacia el exterior. En este sentido, Habegger y Mancila (2006) han planteado que la cartografía social constituye "un proceso democrático de construcción de conocimiento a través de la transcripción de la experiencia de los lugares no nombrados, donde los miembros de la comunidad analizan colectivamente los problemas sociales, en un esfuerzo por comprenderlos y solucionarlos" (Habegger \& Mancila, 2006, p. 6). De aquí deriva nuestra comprensión de que la cartografía social acude al reconocimiento de las formas en que los individuos establecen sus relaciones interpersonales y con el territorio, las cuales pueden plasmarse en el mapa como objeto de conocimiento, además que, según plantea Oslender: "Nos permite interpretar distintas perspectivas y representaciones del espacio, así como distintas experiencias y maneras de relacionarse con el mundo" (Oslender, 2017, p. 250).

Es por ello que se realizaron ejercicios colectivos de construcción de mapas, tal y como el que se observa en la figura 1, los cuales fueron clave para una estrategia comprensiva de las experiencias pedagógicas en la escuela, que se fundamenta en la práctica y acción reflexiva del mapa como objeto de conocimiento que habilita la problematización de los territorios habitados por los integrantes de la comunidad educativa. Más que un fin en sí mismo, coincidimos en que el proceso de mapeamiento constituye un medio adecuado para el intercambio y socialización de saberes y prácticas, identificación de relaciones de poder y disputa de espacios hegemónicos (Ares \& Risler, 2013). En consecuencia, el recurso de la cartografía social en la experiencia de los proyectos realizados en las instituciones educativas de Medellín y el barrio La Gabriela de Bello, partió de comprender que el territorio es una construcción social en la que los maestros y maestras generan las condiciones, prácticas y alternativas para hacer de la escuela un espacio para la formación y las dinámicas transformadoras de la acción pedagógica, enfrentando las tensiones y aprovechando las potencialidades que emergen en las relaciones con los demás miembros de la comunidad educativa. No es posible, ni 
Figura 1. Taller de cartografía social con maestros y maestras.

Institución Educativa Joaquín Vallejo Arbeláez, Medellín

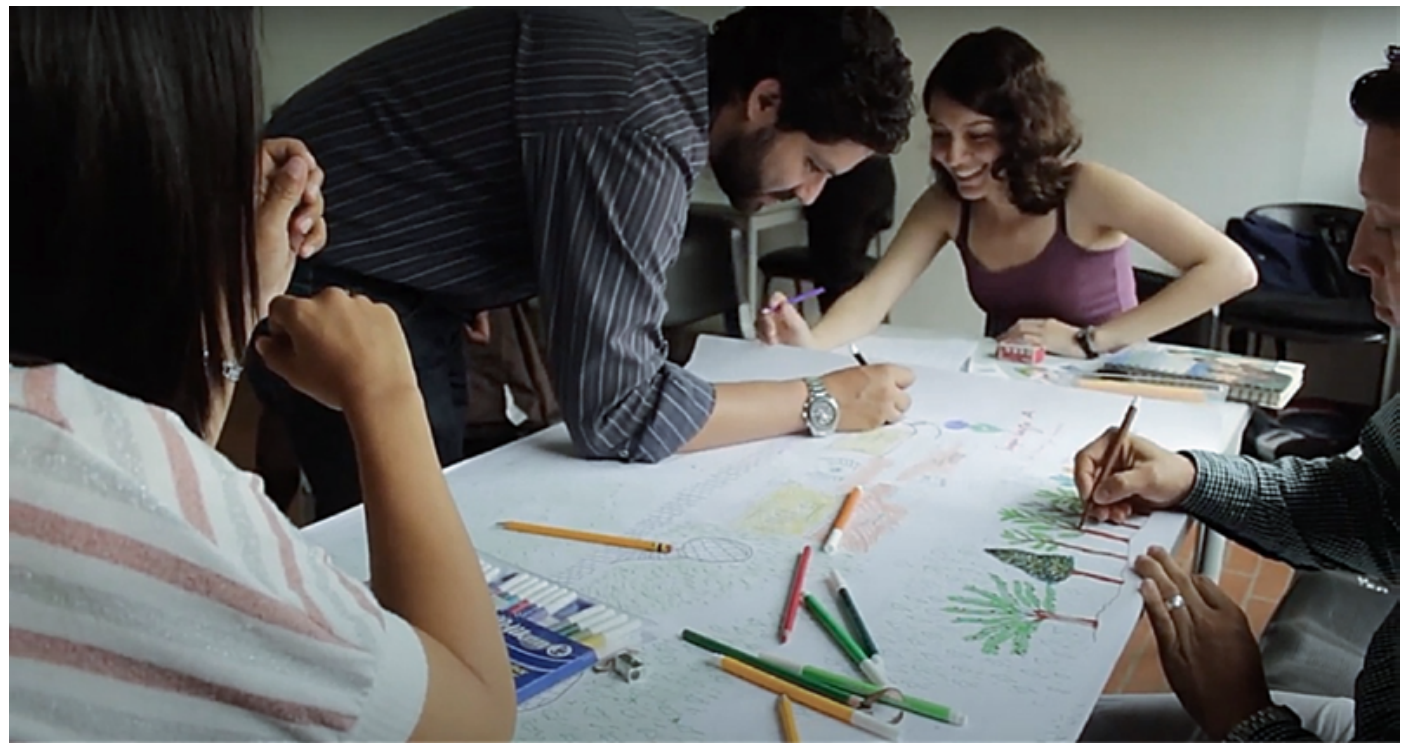

Fuente: proyecto La escuela por dentro (2014).

tampoco deseable, separar al maestro de su escuela-territorio, en tanto que esta es una condición esencial para el desarrollo del proceso de enseñanza-aprendizaje.

El reto metodológico en la aplicación de la cartografía social al ámbito escolar, consiste en el diseño de una estrategia integral y flexible que vaya más allá de la elaboración de mapas, integrando herramientas narrativas diversas, como por ejemplo el video, la fotografía, las piezas sonoras y la escritura de textos, complementando así, las ya tradicionales técnicas de investigación cualitativa. La incursión de las cámaras de video y de fotografía, la grabadora y el ejercicio de escritura con docentes en la cotidianidad de la escuela, permiten que sus voces sean protagonistas durante todo el proceso de investigación que, para nuestro caso, se situó en la exploración de las experiencias pedagógicas. La ruta metodológica de aplicación de la cartografía social para la comprensión de la escuela-territorio, se sintetizó en tres momentos:

1) Concertación y establecimiento de confianza con las instituciones, directivos y docentes. Constituye el punto de partida e implica la convocatoria a que se vinculen como parte activa del proyecto. Este momento requiere del reconocimiento de las dinámicas particulares de las instituciones territorios 44 Especial 
educativas, que suelen ser refractarias a la apertura hacia actores externos. En el proyecto adelantado en Medellín, por ejemplo, se tuvieron varias dificultades en el permiso de ingreso a las instituciones educativas y este primer acercamiento nos permitió entender que la entrada a la escuelaterritorio se viabiliza más fácilmente a través de sus docentes que de sus directivos.

2) Exploración de experiencias pedagógicas desarrolladas en los espacios escolares a partir de visitas de interacción con maestros y alumnos, observación participante de las dinámicas cotidianas, conversaciones informales y entrevistas semiestructuradas con las y los docentes, directivos y personal administrativo, talleres y grupos focales, recorridos de reconocimiento al interior de las escuelas para la identificación conjunta de distintos espacios de encuentro, de ocio o de recuerdos significativos. También se realizaron recorridos territoriales en los sectores que rodean las escuelas, estableciendo conversaciones con algunos vecinos y padres de familia de los y las estudiantes. Esta participación en la cotidianidad de la escuela permite identificar que muchas de las experiencias pedagógicas desplegadas en las aulas, abordan distintas tensiones o potencialidades de la vida en sociedad de los integrantes de la comunidad educativa, además de que no se limitan al ámbito de lo académico, sino que sirven de conexión con las problemáticas ambientales, sociales y culturales, propias de la realidad barrial.

3) Diseño y realización de piezas de visibilización y apropiación social de las experiencias pedagógicas, estructuradas en espacios de reflexión colectiva y en tareas individuales que adelantaban los maestros en sus casas y traían a los encuentros de asesoría con el equipo de investigación. Se trató de un proceso de confección reflexiva de piezas sonoras (a modo de radio-novelas), videos cortos, fotoreportajes y relatos escritos que, compilaban las memorias de los docentes y alumnos sobre aquellas experiencias pedagógicas inscritas en sus recuerdos y transmitidas año tras año a los nuevos grupos de la escuela. Esta instancia es una manera de mapear las experiencias pedagógicas, identificando los lugares donde ocurren, los elementos que se utilizan, las personas que intervienen y los espacios que las hacen posibles; resultó ser el dispositivo clave para la reflexión sobre las acciones pedagógicas en clave territorial. El dibujo a mano alzada de la institución, de sus elementos constitutivos, de sus espacios significativos y de sus eventos, abre unas posibilidades de diálogo inéditas entre maestros, alumnos y familias sobre los factores sociales, ambientales, culturales, políticos 
y administrativos que impactan la escuela, transformándola, condicionándola o fortaleciéndola en su interior y en relación con sus contextos circundantes.

\section{Experiencias pedagógicas $\mathrm{y}$ territorio}

Las instituciones educativas pueden ser vistas como escenarios donde convergen diversas maneras de ver el mundo. La comunidad educativa que la habita lleva consigo saberes, prácticas y discursos que inciden en la convivencia y en la aceptación de la norma. Además de esto, en el caso de las instituciones educativas abordadas, su localización en territorios donde se expresan múltiples problemáticas sociales como son el conflicto armado, el tráfico de drogas, la pobreza, la privación de derechos, la carencia de servicios públicos, la contaminación del agua y el aire, entre otras, marcan significativamente la vida de quienes son parte de la comunidad educativa. Muchos de los maestros y maestras aducen que dichos conflictos socioterritoriales se trasladan a la escuela y a sus aulas, enfrentándolos a situaciones tensionantes para las que no tienen muchas herramientas a la mano, al no obedecer estrictamente al ámbito académico.

Como resultado de las estrategias desplegadas por los maestros para dirimir los conflictos y favorecer la permanencia estudiantil, así como respecto a su intención de aportar desde los aprendizajes de la vida escolar a la gestión de los conflictos de la vida barrial, resultó el aporte a la consolidación de territorios incluyentes y democráticos, especialmente desde lo que hemos denominado experiencias pedagógicas. Esto es, un conjunto de prácticas movilizadas desde las aulas y, en general, desde las inmediaciones escolares, las cuales se van sedimentando año tras año, recogiendo aprendizajes que las renuevan y fortalecen su capacidad transformadora y de incidencia territorial. Es precisamente en estas experiencias pedagógicas que se nutre la relación entre escuela y territorio, poniendo de presente la interdependencia entre el espacio habitado y las relaciones de quienes lo habitan, en este caso, los integrantes de la comunidad educativa. Además, el concepto de escuela-territorio pone de presente las territorialidades que convergen en la escuela, es decir, las formas específicas de apropiar y resignificar el espacio propias de maestros, estudiantes y familias. Dicha territorialidad no se refiere al espacio físico ocupado por la escuela y sus zonas circundantes, sino que refiere a la emergencia de las relaciones sociales, al entramado de acciones, prácticas y discursos que dan legibilidad y reconocimiento a la escuela posicionándole de manera precisa en los distintos actores del territorio. Según señala Echeverría y Rincón (2000), territorio y territorialidad deben ser entendidos en una relación de interdependencia, están imbricados y uno forma parte del otro, configurando una territarios 44 Especial 
Figura 2. Taller de cartografía social. Institución Educativa Lola González, Medellín

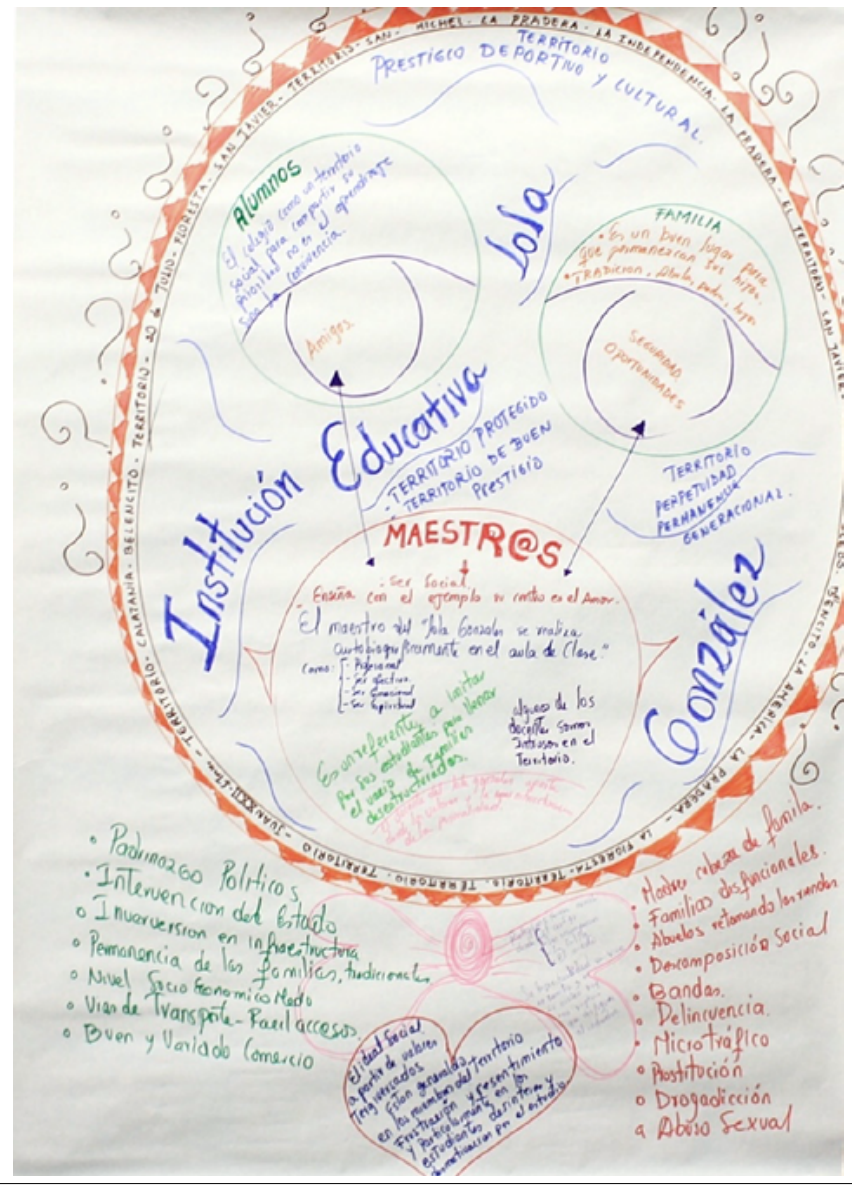

Fuente: proyecto La escuela por dentro (2014).

tersitarias 44 Especial relación dialéctica en la que mientras los sujetos marcan, habitan, transforman y se apropian del territorio, lo van reorganizando de acuerdo a la forma como ellos se relacionan entre sí y al interior del territorio mismo; a su vez, tal territorio afecta y transforma a los sujetos que lo habitan. Según muestra la figura 2, en el caso de la escuela pública Lola González, las relaciones entre alumnos y maestros tienen expresiones territoriales concretas que la configuran como un entorno protector y de permanencia, en tensión con territorialidades externas que implican riesgo o amenazas latentes.

En concordancia con lo anterior, se identificaron las instituciones educativas desde el lugar de los maestros y maestras, como una entidad que está siendo organizada, transformada y marcada por sus acciones, por las estrategias y actividades que se despliegan para desenvolver el saber pedagógico que está vinculado con los sentidos, miedos, percepciones y expectativas del ser maestro. Tal y como lo afirma el pedagogo Carlos Skliar: "Un sujeto de la experiencia pensado como un territorio de paso, como una superficie de sensibilidad en la que algo pasa y deja una huella, una marca, un rastro, una herida" (Skliar, 2007, p. 17). En tal sentido, no solo la dimensión física de la institución educativa es incidida por las acciones del maestro, también lo es su mismo cuerpo cuando se convierte simbólicamente en territorio, en un espacio donde ocurren, se devuelven y se reflejan las anécdotas y vivencias de la vida escolar. Nuestra comprensión de la escuela como territorio implica, por lo tanto, abordarla desde tres ámbitos socioespaciales particulares: (1) las dinámicas generadas por las prácticas de los sujetos que la habitan y la rodean, sus comprensiones y vivencias 
del mundo, la diversidad de sus memorias, intereses y expectativas; (2) las dinámicas propias de la escuela como institución educativa; y (3) las dinámicas que movilizan la relación de la escuela con los entornos barriales en los que se emplaza. La escuela como territorio puede entenderse como una construcción social en la que los maestros y maestras generan las condiciones, prácticas y alternativas para convertirla en un espacio para la formación y el despliegue de sus prácticas y saber pedagógico, de cara a las tensiones y potencialidades propias de las relaciones de los integrantes de la comunidad educativa con sus realidades socioculturales.

En relación con el primero de los ámbitos socioespaciales, se encuentra que lo sucedido a los maestros y maestras cuando desarrollan las funciones escolares planeadas o espontáneas, provoca cambios en sus cuerpos y afecta sus subjetividades, creando marcas que van definiendo su condición de ser maestros. En el segundo ámbito, se halla que son esenciales las prácticas pedagógicas y las relaciones que acontecen a los distintos integrantes de la comunidad educativa (estudiantes, maestros, directivos, personal administrativo, del aseo o la vigilancia), en los distintos lugares de la escuela, sean los salones como primer escenario del proceso de formación, pero también los corredores, las canchas, las escaleras, las áreas verdes $\mathrm{y}$, en general, todos aquellos espacios físicos que se reconocen como parte de la institución y que habilitan el encuentro y el reconocimiento mutuo. Por último, en relación con el tercer ámbito socioespacial, se detectó que es incoherente limitar la comprensión de la escuela a su interioridad, sin el reconocimiento de que lo que está sucediendo dentro de la institución se encuentra ligado a las condiciones sociales, ambientales e históricas de la localidad o el barrio en el que se encuentra ubicada.

\section{Aproximación a las experiencias pedagógicas desde las voces de maestros y maestras en las instituciones educativas}

De acuerdo con la pedagoga Nuria Pérez (2010), investigar la experiencia pedagógica es, ante todo, estudiar lo educativo en tanto que vivido y acercarse a lo que personas concretas viven y experimentan en sí mismas. En ese marco, Jorge Larrosa (2009) plantea que una experiencia es eso que me pasa, asociado a un acontecimiento contenido en el exterior que, en un movimiento de devolución, tiene lugar en las palabras, los proyectos, las representaciones, sentimientos, intenciones o saberes de alguien. Se trata de un movimiento de ida y vuelta que sale de sí mismo hacia afuera, hacia el encuentro con eso que pasa y retorna generando efectos en ese alguien, en lo que es, lo que siente, sabe y quiere. Para los maestros y maestras, la experiencia se hace pedagógica en clave con los significados y el sentido de lo vivido en vinculación territarios 44 Especial 
con las maneras en que sucede la relación y el encuentro con sus estudiantes, pares docentes, familias, directivos, pero también con los escenarios de las instituciones educativas y con los procesos históricos y sociales de los barrios donde se encuentran ubicadas. En este apartado traemos algunas voces de los y las docentes que participaron de los dos proyectos de investigación, observando la manera en que sus experiencias pedagógicas nos hablan de territorios y territorialidades.

En la cartografía de las experiencias pedagógicas y los mapeos colectivos, los y las docentes referenciaron su escuela como

Un territorio con fondo, pero a veces solo la concebimos como algo superficial. Mirar solo la forma de las cosas y no su fondo que impide que nuestra mente llegue más lejos de lo que nuestros ojos puedan ver. La escuela es un territorio donde se encuentran todo tipo de seres, sentimientos, saberes y pasiones, unos concebidos, otros impuestos, otros asimilados a voluntad o por fuerza (Taller con maestros y maestras de la Institución Educativa Lola González, comunicación personal, enero del 2014).

Desde esta perspectiva el territorio modifica la práctica pedagógica si se tiene en cuenta que todas aquellas particularidades de la comunidad educativa, sus formas de ser-estar, necesidades y realidades sociales e históricas barriales, se constituyen en factores que determinan el proceso de enseñanza-aprendizaje, y que en muchos casos se convierten en preguntas y retos que la escuela asume para propiciar estrategias y acciones formativas que van enriqueciendo la práctica educativa. Este vínculo apunta a leer y comprender el escenario del aula en clave del reconocimiento de las situaciones barriales. Tanto en la figura 3, como en el testimonio siguiente, derivados de un taller con docentes de la Institución Educativa La Gabriela, se identificaron varios factores del entorno que impactan la institución y la dinámica de su comunidad educativa, de forma que los y las docentes manifiestan que:

Lo que más se visualiza en el barrio es el sector comercial (locales comerciales), y el único espacio de encuentro y de esparcimiento es la cancha. Hicimos unión de La Gabriela con La Madera, porque este se convierte en espacio de encuentro para los niños y niñas estudiantes de la escuela, quienes tienen que hacer un amplio recorrido del barrio a la estación para llegar hasta allí. Se encuentran problemas en la comunidad: conflictos familiares (esto es un diario vivir y sentir en nuestros estudiantes, relaciones familiares complejas); conflicto armado, es difícil señalizarlo porque se encuentra en toda la comunidad (estudiantes utilizados para transportes armas y drogas); además, falta de espacios públicos y de encuentro donde los niños y sus familias puedan tener posibilidad de esparcimiento (Taller con docentes de la 
Figura 3. Taller de cartografía social. Institución Educativa La Gabriela, Bello

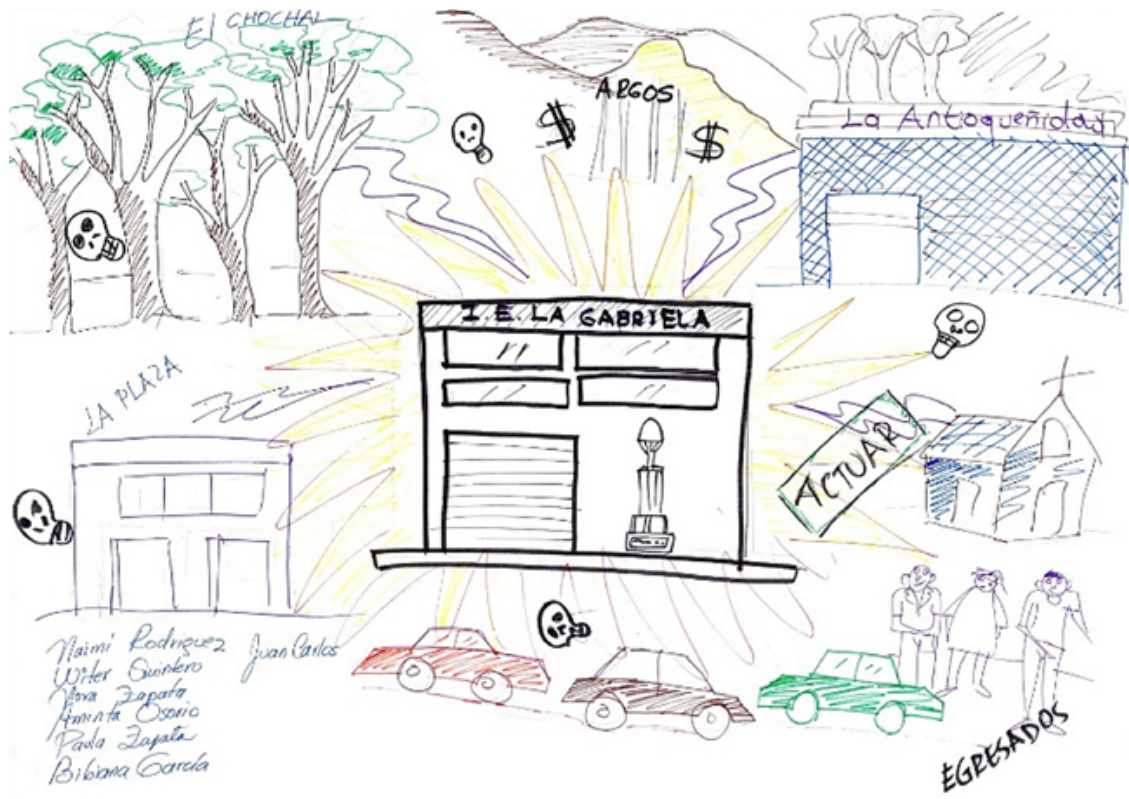

Fuente: proyecto Apropiación social del territorio y educación para la sostenibilidad (2016).

Institución Educativa La Gabriela, Bello, comunicación personal, enero del 2016).

Estas dinámicas y conflictos externos a la escuela, que en múltiples ocasiones se trasladan a su interior, son abordados y visibilizados a través de experiencias pedagógicas situadas en el aula de clase, haciendo de este espacio parte constitutiva del proceso de formación y un escenario activo en el aprendizaje de los estudiantes. Para la maestra Luz Elena Acevedo, de la Institución Educativa Lola González de la Comuna 13 en Medellín, su propuesta educativa denominada "Ecoimaginarios" es una estrategia para la formación de líderes y para desencializar, a través del arte y la reflexión en torno al ambiente, las cotidianidades barriales que han sido afectadas por el conflicto armado, las vivencias de su salón de clase y los conflictos en la convivencia de la institución educativa. Así lo manifiesta la maestra:

Ecoimaginarios se fundamenta en pensar en los imaginarios de una comunidad que ha estado atravesada por esas diversas formas de violencia, porque creo que la Comuna 13, muy particularmente, ha sido una comunidad que ha estado atravesada, no solamente por la violencia implícita territorios 44 Especial 


\section{territorias 44 Especial}

de su propia realidad, sino además por la intervención militar, del Estado. [...] Si el arte no sirve para transformar esto, estas formas de pensamiento, esta forma de indiferencia en el propio entorno, entonces el arte no tiene sentido en la escuela [...] empezamos con los chicos a reflexionar sobre eso, sobre ese espacio que ellos habitaban y sobre el cual consideraban que no había que darle una mirada diferente. [...] En Ecoimaginario se empiezan a hacer visibles una cantidad de temas que yo digo no le hemos dado nombre en la escuela, [esta] debería ser el lugar que las debería nombrar, hacer visibles, no para juzgarlas, no para señalarlas, sino por el contrario para entenderlas. Entonces, cuando la escuela hace esa reflexión, empieza a sentirse apoderada: la sexualidad, las formas de poder, la violencia, empiezan a entrar a su manera a la escuela, entonces lo que tenemos que hacer es eso: hagámoslo visible acá (Institución Educativa Lola González, Medellín, comunicación personal, febrero del 2014).

Esa misma clave reflexiva se hace presente en la experiencia "Prácticas corporales recreativas de los habitantes de la Comuna Uno", liderada por el profesor Marco Fidel Gómez, de la Institución Educativa María de los Ángeles Cano, en la que da cuenta de cómo la relación escuela-maestro-estudiante-territorio expresada en la figura 4, contribuye a que la escuela trascienda su espacio físico y se proyecte como eje articulador del territorio en el cual se ubica. Esta experiencia pedagógica toma como referente de indagación a los espacios recreativos de la Comuna 1 de la ciudad de Medellín y a los distintos modos de habitarlos. A través de reflexiones y puesta en común de las percepciones de los estudiantes frente a dichos espacios, el profesor Marco Fidel motiva a sus estudiantes a que realicen recorridos, hagan entrevistas, tomen fotografías e indaguen en otras fuentes de datos sobre su territorio, con el objetivo de construir un acervo de conocimiento amplio que les permita aprender desde su barrio y descubrir cómo este es vivido y, cómo son las maneras en las que los habitantes disfrutan y recrean escenarios de juego, ocio y recreación.

Según puntualiza el profesor Marco Fidel, esta experiencia pedagógica desarrollada como parte de la formación media técnica en recreación busca:

[...] darle un giro a la mirada y a la forma de hacer las medias técnicas, que también pueden enfocarse en tener espacios para pensar, para reflexionar; que en ese escenario de la recreación, cómo se piensa el espacio, cómo se piensa la vida y otros conceptos que encierran el tema de la recreación [...] Un asunto desde la reflexión, política también, y que tiene que ver con la mirada a la comunidad, cómo se puede transformar, qué está pasando [...] (Comunicación personal, febrero del 2014). 
Figura 4. Taller de cartografía social con estudiantes. Institución Educativa María de los Ángeles Cano, Medellín

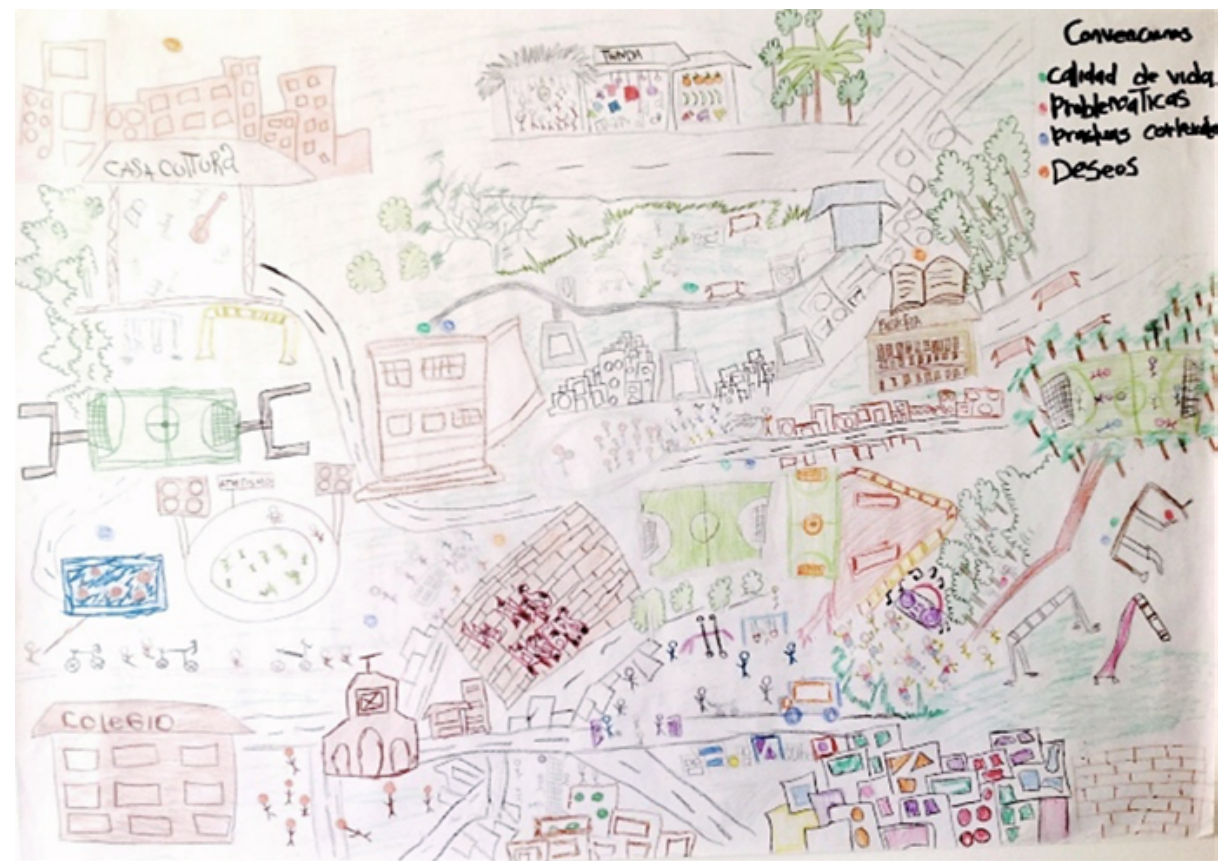

Fuente: Proyecto La escuela por dentro (2014).

A lo cual una estudiante complementa,

Antes de salir al territorio, hacemos un debate sobre lo que vamos a ir a buscar afuera. Si vamos a ir a buscar espacios recreativos, hablamos de esos espacios, luego salimos, vemos cómo se viven en la comunidad y cómo nosotros creemos y los conceptos nos dicen que son. Es un proceso que empieza desde el aula con lo teórico y luego salir a mirar afuera lo práctico (Taller de cartografía social Institución Educativa María de los Ángeles
Cano, Medellín, comunicación personal, febrero del 2014).

La figura del maestro-en-el-territorio indaga por aquello que los educandos viven y experimentan, buscando desnaturalizar conceptos incorporados de manera rutinaria en la vida diaria y permitiendo cuestionar el entorno en que habitan. Así lo plantea la profesora Beatriz Elena Álvarez del Centro Educativo Media Luna del corregimiento de Santa Elena de Medellín: "La comunidad se convierte en un aula abierta, donde nos brindan todos territorios 44 Especial 
sus saberes y los niños pueden compartir; $\mathrm{y}$ desde ese aspecto todos formamos un diálogo de saberes donde la escuela apoya a la comunidad y la comunidad incursiona en la escuela" (Centro Educativo Media Luna, comunicación personal, marzo del 2014).

En varias de las búsquedas y apuestas formativas se puede reconocer que el espacio, es tanto una construcción como un elemento creador y dispositivo esencial en el proceso de enseñanza-aprendizaje. Esto es visible en el hecho de que un espacio como el salón de clases hace parte constitutiva del proceso de formación y es un espacio activo en el aprendizaje de los niños y niñas, jugando un papel importante para lograr la adquisición del conocimiento y las dimensiones que en términos emocionales, sociales y afectivos buscan potenciar los maestros y maestras. Maestros como César Londoño, de la Institución Educativa La Candelaria en la Comuna 1 de Medellín, movilizan en los niños y niñas de menores grados la pregunta por su entorno a través del ejercicio de imaginar, escribir y dibujar. Su experiencia pedagógica denominada los "Bolsilibros", parte de la lectura de un breve cuento que sirve como referencia para que los estudiantes recreen una historia de su comuna,

tersitarias 44 Especial invitando a uno de sus personajes favoritos de la cultura, de la música, sus ídolos a que le muestren el barrio Santo Domingo Savio, los sitios donde los llevarían. Ellos plantean que los llevarían a la Biblioteca España, al metrocable Santo Domingo, es decir, muestran los sitios más tradicionales que tiene el barrio [...] Inicialmente, se maneja la redacción, identificar procesos semánticos y semióticos para que una palabra ajuste con la otra y que el texto dé cuenta de la historia que se relata (Institución Educativa La Candelaria, comunicación personal, febrero del 2014).

En esa perspectiva, encontramos en las instituciones educativas de los corregimientos de Medellín algunas apuestas pedagógicas interesantes en su propósito de articular el componente curricular al territorio y a las particularidades que lo constituyen, tales como las condiciones ambientales y la riqueza ecosistémica, las tradiciones y prácticas socioculturales de sus habitantes. La experiencia del maestro de ciencias naturales, John Mario López, en la Institución Educativa Héctor Rogelio del corregimiento San Sebastián de Palmitas, se desarrolla en torno al Plan Ambiental Escolar (PRAE), con el propósito de generar en sus estudiantes sentidos identitarios de pertenencia y apropiación por el corregimiento. Para ello, la huerta escolar se convirtió en la estrategia metodológica para aplicar no solo los contenidos académicos de la materia, sino en un espacio para generar diálogos que además de dar a conocer las características ambientales del corregimiento, tales como el suelo, la fauna y la flora, invitan a los y las estudiantes al desarrollo de 
acciones concretas como el cultivo de hortalizas y plantas medicinales, que históricamente han sido sembradas y usadas por las familias del corregimiento. Según afirman:

Trabajo con la huerta, donde concientizamos a los estudiantes para que ellos activen ese amor por la tierra, por su tierra y por la productividad del corregimiento. Hemos tenido entonces huertas de hortalizas y de plantas medicinales que se han convertido en la posibilidad de estimular y fortalecer la identidad y la apropiación de los niños, niñas y jóvenes por su territorio [...] Estos mismos estudiantes son los que luego van a tomar su lugar en el corregimiento como líderes ambientales, jóvenes que actualmente se encuentran en la red juvenil ambiental, de quienes se espera vayan ocupando un rol de representatividad en el corregimiento, que les permitirá ser los creadores de alternativas de solución para las afectaciones y realidades ambientales (Institución Educativa Héctor Rogelio, San Sebastián de Palmitas, comunicación personal, marzo del 2014).

Este tipo de experiencias logran un efecto reflexivo y de aprendizaje en el maestro, también que los estudiantes resignifiquen esos lugares que para ellos son importantes en su barrio, comuna o corregimiento y, además, logren ligar el conocimiento escolar con los conocimientos populares y la memoria social de los territorios en los que se emplaza la escuela. Con ello, la posibilidad de habitar de una manera distinta los territorios se dinamiza desde las estrategias de maestros y maestras que apuntan por una educación que se deja sorprender por lo que pasa y lo que les pasa a los miembros de la comunidad educativa para descubrir nuevas prácticas y sentidos educativos. En últimas, se apunta a una educación más incluyente y anclada a las realidades locales de quienes habitan la escuela, avanzando en una propuesta de pedagogía del territorio que recoge éticamente la afirmación de la pluralidad epistemológica propia de nuestra nación colombiana.

\section{Conclusiones}

La escuela, en clave de una pedagogía del territorio, se convierte en un escenario propicio para el despliegue de formas de enseñanza-aprendizaje en los que las memorias y las formas de ver el mundo de quienes conforman la comunidad educativa, alimentan la creatividad para la gestión de las tensiones sociales, políticas, culturales y ambientales circundantes. De esta manera, la escuela trasciende su condición de infraestructura en la que se transmiten y reproducen conocimientos, produciendo un espacio propicio para la reflexión política y el compromiso social. La forma en que las experiencias pedagógicas son creadas y reproducidas en los distintos escenarios escolares y barriales, proporciona elementos valiosos para tersitorias 44 Especial 


\section{tersitorias} 44 Especial entender el territorio como factor pedagógico útil para la construcción de ciudadanías incluyentes y reflexivas, además de aproximar a las instituciones educativas al reconocimiento de la diversidad epistémica propia de los colectivos sociales, comunidades étnicas o grupos diferenciales con los que interactúa. Estas iniciativas se articulan al llamado que se ha venido haciendo desde distintos lugares de Latinoamérica, sobre la necesidad de promover modelos pedagógicos interculturales útiles para la recuperación de los conocimientos locales y el fortalecimiento de las experiencias comunitarias (Bertely et al., 2015).

En los proyectos de investigación descritos en este trabajo, la cartografía social demostró su potencia como estrategia para la investigación educativa que nutrió y preparó escenarios que sondearon las cuestiones pedagógicas de las experiencias. Como forma de producción de conocimientos, más allá de una simple herramienta metodológica que es funcional para el re-conocimiento del territorio que adquiere una connotación política que convoca las voces de la comunidad para visibilizar las tensiones y potencialidades territoriales y proyectarlas de forma propositiva a los modos de habitar, pensar y vivir los espacios. Con los ejercicios de cartografía social adelantados en las instituciones educativas, se revelaron tres ámbitos socioespaciales significativos: (1) el cuerpo y las subjetividades del docente que se incorporan y transforman desde las vivencias del escenario escolar, (2) la escuela por dentro, entendida como el espacio discursivo en el que emergen las experiencias pedagógicas desde las distintas inmediaciones de la escuela y, (3) la escuela en el territorio con sus múltiples relaciones sociales, políticas, culturales y ambientales con los barrios en los que se emplaza.

Conforme a lo anterior, se pueden derivar de la escuela-territorio interrogantes pedagógicos y rutas de enseñanza y aprendizaje reflexivos y propicios para conectar lo que sucede en la escuela con las realidades vividas por los integrantes de la comunidad educativa, buscando con ello un proceso de formación crítico y transformador, tal y como lo plantea Giroux (2013): "El rol de una educación crítica no es entrenar a los estudiantes solamente para trabajar, sino además educarlos para cuestionar críticamente las instituciones, las políticas y los valores que dan forma a sus vidas, las relaciones con los demás y una infinidad de vínculos con el mundo en general" (Giroux, 2013, p. 17). En estas condiciones, los procesos de construcción de conocimiento vinculados con las vivencias y situaciones cotidianas de los y las estudiantes, habilitan la creación de espacios colectivos para descubrir, compartir, debatir y reconstruir nuevos significados y proponer horizontes alternativos sobre las realidades y aconteceres de sus localidades. Según lo encontrado en las experiencias pedagógicas descritas en las instituciones educativas 
de Medellín y Bello, en el contexto de ciudades con problemáticas tan complejas de violencia, desigualdad e inequidad, la creación de dichos espacios colectivos en la escuela permite que la denominada pedagogía del territorio sea una apertura para afianzar la responsabilidad política de la educación, que habilite diálogos amplios y promueva la recuperación de confianza entre los actores externos y los integrantes de la comunidad educativa.

\section{Referencias}

Ares, P., \& Risler, J. (2013). Manual de mapeo participativo. Tinta Limón.

Acselrad, H., \& Régis, L. (2008). Disputas territoriais e disputas cartográficas. En H. Ascelard (Org.), Cartografias Sociais e Território (pp. 13-44). IPPUR/UFRJ.

Barragán, D., Cruz, A., \& Sánchez, N. (2020). Cartografía social, usos y sospechas en el campo de la educación. Utopía y Praxis Latinoamericana, 25(89), 179-198. http://doi. org/10.5281/zenodo.3740109

Barragán, D. (2016). Cartografía social pedagógica: entre teoría y metodología. Revista Colombiana de Educación, (70), 247-285. https://www.redalyc. org/pdf/4136/413644492012.pdf

Bertely, M., Martínez, M., \& Muñoz, R. (2015). Autonomía, territorio y educación intercultural. Actores locales y experiencias comunitarias latinoamericanas. Desacatos, (48), 6-11. http:// www.scielo.org. $\mathrm{mx} / \mathrm{pdf} /$ desacatos/ n48/n48al.pdf

Echeverría, M., \& Rincón, A. (2000). Ciudad de territorialidades: polémicas de Medellín. Centro de Estudios del Hábitat Popular, Universidad Nacional de Colombia.

Freire, P. (2012). Pedagogía de la autonomía. Saberes necesarios para la práctica educativa. Siglo XXI Editores.

Giroux, H. (2013). La pedagogía crítica en tiempos oscuros. Praxis Educativa, 17(1), 13-26. http://170.210.120.129/index.php/ praxis/article/view/1648/1668

Grupo Estudios del Territorio. (2014). La escuela por dentro. Instituto de Estudios Regionales, Universidad de Antioquia.

Grupo Estudios del Territorio. (2016). Apropiación social del territorio y educación para la sostenibilidad sector La Gabriela. Instituto de Estudios Regionales, Universidad de Antioquia.

Habegger, S., \& Mancila, I. (2006). El poder de la Cartografía Social en las prácticas contrahegemónicas o la Cartografía Social como estrategia para diagnosticar nuestro territorio. Revista Araciega, 14, 1-10. https:// cutt.ly/Exxhaxq

Larrosa, J. (2009). Experiencia y alteridad en educación. En J. Skliar \& J. Larrosa (Comp.), Experiencia y alteridad en educación (pp. 13-45). Homo Sapiens Ediciones. territarios 44 Especial 
López, C. (2018). La cartografía social como herramienta educativa. Revista Scientific, 3(10), 232-247. https://dialnet.unirioja.es/servlet/ articulo? codigo $=7011992$

Montoya, V., García, A., \& Ospina, C. (2014). Andar dibujando y dibujar andando. Cartografía social y producción colectiva de conocimientos. Nómadas, (40), 191205. https://www.redalyc.org/ pdf/1051/105131005013.pdf

Oslender, U. (2017). Ontología relacional y cartografía social: ¿Hacia un contra-mapeo emancipador, o ilusión contrahegemónica?. Revista Tabula Rasa, (26), 247-263.

Parra, J. (2020). La educación para la paz en el contexto de las ciencias sociales y la formación ciudadana en la escuela: aportes de la cartografía social [Tesis doctoral en Educación].
Universidad de Antioquia. https:// bit.ly/382FKYz

Patiño, O. (2017). El potencial didáctico de la cartografía social en la enseñanza de la geografía y las problemáticas socioespaciales [Tesis de maestría]. Universidad de Antioquia. https:// bit.ly/2wWrscq

Pérez, N., \& Contreras J. (2010). La experiencia y la investigación educativa. En J. Contreras \& N. Pérez (Comp.), Investigar la experiencia educativa (pp. 885-892). Ediciones Morata.

Skliar, C. (2007). La educación (que es) del otro. Argumentos y desierto de argumentos pedagógicos. Noveduc.

Uribe, C., Donoso, D., \& Ramírez, A. (2017). De la cartografía social a la comprensión de los contextos socioeducativos. Aletheia, 9(2), 74-93. http://dx.doi.org/10.11600/21450 366.9.2aletheia.74.93 